\title{
Les éleveurs et l'estive : pour une approche compréhensive des pratiques pastorales
}

\author{
Corinne Eychenne \\ Géographe, Université Toulouse 2 - Le Mirail, UMR Dynamiques rurales, 5 allées Antonio Machado, 31058 Toulouse cedex, France
}

\begin{abstract}
Scruter les nouvelles relations entre éleveurs et ressources pastorales « communes » en accordant une attention équivalente à la construction de nouvelles identités sociales, au rapport à l'herbe des animaux et à l'évolution même du système complexe de l'estive, telle est l'ambition de la recherche que propose Corinne Eychenne. L'article donne corps à l'idée que, même dans la solitude du jeune chercheur sur son terrain et en-dehors d'un programme interdisciplinaire, la posture interdisciplinaire ou d'indiscipline a une valeur innovante pour traiter des rapports sociétés/natures. D'où l'intérêt que manifeste dans son commentaire, le sociologue C. Compagnone : il y relève à la fois des avancées proprement sociologiques et le "souci de la matérialité des choses de l'approche géographique qui échappe parfois à la sociologie ».
\end{abstract}

La Rédaction

\section{Mots-clés : pastoralisme ; montagne; géographie ; approche compréhensive ; Pyrénées}

\section{Keywords: pastoralism; mountain; geography; understanding approach; Pyrénées}

\begin{abstract}
Résumé - Dans les Pyrénées ariégeoises, la restructuration de l'élevage de montagne et du pastoralisme est à l'origine d'une profonde modification des relations que les éleveurs entretiennent avec les territoires d'altitude. La compréhension du sens endogène des pratiques des éleveurs transhumants permet de mettre au jour l'émergence et la confrontation de deux référentiels techniques et symboliques sur la montagne. Alors que l'estive reste le principe organisateur et identitaire des éleveurs « usagers » de haute montagne, elle tend à être envisagée comme une opportunité économique parmi d'autres par les éleveurs " étrangers » pratiquant une transhumance de proximité. L'attachement aux droits d'usage, seuls garants de la légitimité des hommes et des bêtes sur la "montagne ", permet alors aux premiers, même minoritaires, de garder le contrôle des modalités de gestion de la ressource pastorale.
\end{abstract}

\begin{abstract}
Cattle farmers and mountain summer pastures: a comprehensive approach to pastoral practices. In the Ariège department (French Pyrénées), the restructuring of mountain cattle farming and of pastoralism has brought about profound changes in the relations cattle farmers entertain with the high mountain pastureland. Gaining insight into the endogenous meaning of the practices of cattle breeders traditionally using these summer pastures allows the highlighting of the emergence and confrontation of two technical and symbolic bodies of references on the mountain. While the summer pastures remain the organizing and identitary principle for the mountain farmers who are the traditional "users" of the high mountain area, other "non-local" farmers from lower lying areas who practise short distance transhumance view these pastures as one among other economic opportunities. Strong attachment to customary rights, the sole guarantee for people's and animals' legitimacy to use the "high mountain", enables these high mountain farmers, even though a minority, to keep control over the management methods of grazing resources.
\end{abstract}

La question pastorale est un « vieil » objet géographique, qui a longtemps été au cœur de l'analyse des relations que les sociétés humaines entretiennent avec les espaces montagnards. Depuis une vingtaine d'années, pourtant, les géographes, notamment les ruralistes, se sont détournés de cette question, que les agronomes ou les environnementalistes continuent d'explorer. Dans

Auteur correspondant : corinne.eychenne@univ-tlse2.fr ce cadre, l'effort de recherche porte prioritairement sur les conséquences paysagères et environnementales de l'évolution des pratiques des éleveurs. Les dynamiques actuelles y sont le plus souvent envisagées comme des dégradations du système agro-sylvo-pastoral traditionnel. Cet article propose de rompre avec ce paradigme du déclin et de réexaminer la question pastorale dans sa modernité. Les éleveurs ont changé, et avec eux les 
modalités de leurs relations à l'espace pastoral, qui ne peuvent plus être envisagées en référence à une norme vieille d'un siècle et demi.

L'ambition affichée de porter un regard neuf sur le pastoralisme ariégeois suppose de changer de méthode. La posture retenue ici vise à renouer avec la tradition d'une géographie interdisciplinaire liant intrinsèquement les dimensions matérielles et sociales des objets étudiés. Elle emprunte néanmoins aux disciplines voisines en affirmant que la compréhension des rapports que les éleveurs entretiennent avec l'estive doit passer par une analyse $\mathrm{du}$ sens endogène qu' $^{\prime} \mathrm{ils}$ attribuent à leurs pratiques, une mise au jour de leurs raisons de faire. Cette entrée par les hommes eux-mêmes est rendue incontournable par les recompositions de l'élevage transhumant en Ariège, qui ont ouvert l'accès aux «montagnes » à de nouvelles catégories d'éleveurs. Il convient donc d'étudier la confrontation des référentiels techniques et symboliques des éleveurs transhumants dans leur définition d'un rapport actualisé non seulement à la ressource, mais également aux dimensions sociales et identitaires de la pratique pastorale.

\section{Pour une approche compréhensive des relations entre les éleveurs et l'estive}

Le corpus de travaux consacrés à l'élevage pyrénéen est si abondant qu'il convient de préciser en quoi une nouvelle approche se justifie aujourd'hui. Il n'est pas question ici de réaliser une analyse poussée de ces différents travaux ${ }^{1}$, mais de mettre en évidence quelques grandes étapes du regard porté par la recherche sur la question du pastoralisme pyrénéen. Dans la première période, les monographies régionales (Cavaillès, 1931; Chevalier, 1956) offrent une description précieuse des modalités traditionnelles de gestion de l'espace par les communautés montagnardes, mais elles prennent surtout acte de la fin d'un monde : le système agro-sylvo-pastoral, érigé en modèle d'une relation harmonieuse entre les hommes et la montagne, n'a pas survécu à la crise démographique, économique et sociale de la fin du XIX ${ }^{e}$ siècle. La gestion des ressources pastorales est alors envisagée à travers les notions de déclin, d'archaïsme et de résistance au changement, avec en toile de fond l'abandon des pratiques collectives minutieusement réglées. Cette approche en termes de décomposition d'un système traditionnel considéré comme optimal va marquer pour longtemps le regard porté sur l'agriculture pyrénéenne.

Ainsi en est-il du deuxième temps fort de la recherche articulé autour du programme interdisciplinaire Élevage pyrénéen ${ }^{2}$, réunissant agronomes, géographes et professionnels, pour traiter des conditions de reproductibilité

\footnotetext{
1 Analyse réalisée par ailleurs (Eychenne, 2003).

2 Programme Élevage pyrénéen, financé par la DGRST entre 1976 et 1982, sous la direction de Jean-Claude Flamant et
}

agronomique et sociale de l'élevage dans les Pyrénées centrales. L'étude fine des systèmes d'élevage (Duru et al., 1979), comme celle des stratégies foncières (Balent et Barrué-Pastor, 1986), concluent à leur tour à une dégradation des pratiques liée au manque de technicité des éleveurs et à la poursuite de stratégies individuelles. De ces travaux ressort l'image d'une société déstructurée, vieillie et désorientée qui n'assure plus les conditions de sa reproduction, pour n'avoir pas su réinventer de nouvelles formes de rapport à l'espace près d'un siècle après les bouleversements qui avaient mis à bas les anciens cadres.

Une troisième étape s'engage à partir des années 1990, avec la montée en puissance des préoccupations agrienvironnementales. Les efforts de la recherche se concentrent sur les zones intermédiaires, où les enjeux sociaux et paysagers paraissent plus marqués qu'en estive (dynamiques de végétation, proximité de l'habitat, foncier privé). L'Inra-SAD de Toulouse poursuit ses travaux autour des effets des pratiques d'élevage sur la durabilité des ressources pastorales et des paysages, principalement sur les fonds de vallée et les zones intermédiaires (Di Pietro et Balent, 1997; Gibon, 1997). Pendant cette période, l'estive est le plus souvent envisagée comme une «boîte noire », sauf dans les travaux du laboratoire Géode sur les liens entre les pratiques pastorales et les dynamiques de végétation sur les espaces d'altitude (Métailié, 1999).

Dans ces approches riches et diversifiées, les éleveurs sont généralement envisagés de manière indirecte à travers l'étude des systèmes d'élevage, l'observation des pratiques pastorales et de leurs effets paysagers ou environnementaux. Les représentations et les raisons d'agir des éleveurs ne sont pas abordées ${ }^{3}$, il s'agit plutôt d'identifier les «bonnes » et les «mauvaises » pratiques en termes de renouvellement de la ressource pastorale et d'entretien du paysage, et l'on voit parfois resurgir dans le discours expert les représentations tenaces liées à l'inertie, l'archaïsme ou l'individualisme des éleveurs pyrénéens pour expliquer les dynamiques paysagères régressives ou la résistance à la réintroduction des grands prédateurs, par exemple.

C'est dans ce contexte qu'est apparue la nécessité d'une nouvelle approche des relations hommes-milieu dans les espaces montagnards, qui s'affranchirait du paradigme de la dégradation des pratiques. Cette posture suppose d'en finir avec le système agro-sylvo-pastoral traditionnel comme étalon des relations société-nature en montagne et d'envisager les pratiques et les représentations actuelles

Georges Bertrand, engageant l'Inra-SAD de Toulouse, l'Institut de géographie de 1'Université de Toulouse-Le Mirail (CIMACNRS), l'ENSAT, l'INERM, le SUACI Pyrénées et la chambre régionale d'agriculture Midi-Pyrénées.

${ }^{3}$ On peut cependant signaler les travaux de Monique BarruéPastor et Valérie Fournié (1996) sur les perceptions de la procédure « article 19 » par les éleveurs ariégeois. 
des éleveurs comme une nouvelle étape dans les recompositions territoriales à l'œuvre dans les Pyrénées. «Une nouvelle posture de recherche est [...] à constituer car il ne s'agit plus seulement de décrire des rapports hommenature déjà élaborés et négociés sur la longue durée [...], mais d'inventer (ou de réinventer) de nouveaux types de négociation des rapports sociétés-nature, de nouvelles pratiques, à partir des techniques et des représentations du milieu naturel contemporain ou à venir » (Mathieu, 1992). Il s'agit de donc de modifier le regard porté sur une catégorie d'acteurs (les éleveurs transhumants) et une catégorie d'espace (l'estive), en renouant avec une géographie qui se réclame à la fois des sciences de la nature et des sciences de la société (ibid.). Bien que l'approche retenue ici vise prioritairement à mettre au jour les dimensions sociales et symboliques du rapport des éleveurs à la montagne, elle comporte également une dimension physique liée au sol, au relief, à la végétation, à la «matière », envisagée à travers ce qu'en disent les éleveurs. Pour répondre à cet objectif, l'interdisciplinarité interne à la géographie est enrichie par une pluridisciplinarité de trajectoire liée à ma formation de zootechnicienne, mais aussi une pluridisciplinarité d'emprunts, notamment à la sociologie, pour développer une approche centrée sur le système de pensée des éleveurs.

En effet, la déconstruction des stéréotypes et la compréhension des nouvelles dynamiques à l'œuvre dans la relation que les éleveurs entretiennent avec les territoires d'altitude suppose le recours à une posture méthodologiquement engagée. Il s'agit de reconnaître une haute valeur heuristique au point de vue des éleveurs, non qu'il soit plus ou moins légitime que celui des autres catégories d'acteurs, mais parce qu'il est négligé, voire dominé par le discours savant, technique et politique. Reconnaître les éleveurs comme des acteurs rationnels prenant des décisions, non seulement dans le contexte spécifique de leur exploitation, mais aussi au sein d'une société locale caractérisée par une histoire, un fonctionnement, un territoire, suppose donc de considérer qu'ils ont « de bonnes raisons de faire ce qu'ils font ». L'étude des pratiques pastorales est donc abordée selon une posture centrée sur la rationalité des éleveurs, largement développée dans la sociologie rurale des années 1980 (Darré, 1985 ; Pharo, 1985 ; Salmona, 1994), mais singulièrement absente des travaux consacrés à la gestion des espaces d'altitude.

Cette approche repose sur une analyse croisée entre représentations et pratiques, centrée sur le discours des éleveurs et la participation active à certaines pratiques fortement professionnalisées, rendue possible par des compétences acquises hors champ disciplinaire ${ }^{4}$. Cet engagement aux côtés des éleveurs, l'attention portée à leurs façons de faire, de (se) penser et de (se) dire

\footnotetext{
${ }^{4}$ Liées à ma position de zootechnicienne et de femme d'éleveur, néanmoins non inséré au champ social étudié au moment de l'étude.
}

permettent d'accéder à une forme de connaissance des relations qu'ils entretiennent avec les espaces pastoraux qui resterait sinon inaccessible. Il ne s'agit pas pour le chercheur de se faire «tribune » ou «porte-voix», mais bien d'affirmer que le rôle de la recherche est aussi de révéler et d'analyser les raisons de faire de groupes socialement dominés.

Pour atteindre ces objectifs, les entretiens semi-directifs approfondis ${ }^{5}$, sous forme de récits de pratiques, sont privilégiés. La connaissance est alors produite par l'analyse du sens que les acteurs donnent eux-mêmes à leurs pratiques. Il s'agit de mettre en évidence les systèmes de valeur, les repères normatifs à partir desquels ils s'orientent et se déterminent. L'analyse des discours vise donc à identifier les différentes normes régissant le rapport des éleveurs à l'estive et à dégager leurs fonctions non seulement opératoires, mais aussi sociales et identitaires (Darré, 1985). D'une manière générale, l'analyse des entretiens cherche moins à obtenir des informations précises sur des faits avérés qu'à comprendre comment les éleveurs conçoivent les choses et justifient, pour eux-mêmes et pour le chercheur, leurs façons d'agir. "Il ne s'agit pas de chercher du caché, de l'inconscient, de l'inattendu, mais plutôt de relever ce qui est tellement manifeste qu'on n'y prend pas garde. Il s'agit d'accumuler du manifeste pour dégager des formes d'organisation de la réalité qui, elles, ne sont pas nécessairement évidentes au premier abord » (Darré et al., 1993). L'enjeu d'une approche compréhensive de la question pastorale est donc de réinterroger les pratiques actuelles en fonction du sens que leur attribuent, ici et maintenant, ceux qui utilisent les pâturages d'altitude.

\section{L'émergence d'une transhumance de proximité : confrontations techniques et rapport à la ressource}

La restructuration de l'élevage pyrénéen est désormais bien connue (Gibon, 1997 ; Eychenne, 2006), ainsi que son entrée dans une logique de marché et son adaptation aux dispositifs publics spécifiques aux zones défavorisées ou relevant des principes généraux de la $\mathrm{PAC}^{6}$. Les estives,

\footnotetext{
${ }^{5}$ Soixante-quatre entretiens semi-directifs effectués auprès d'éleveurs, plus une dizaine d'entretiens auprès de pâtres, d'élus locaux, de représentants des administrations, de techniciens, etc., entre avril 1998 et août 2001, complétés de phases d'observation directe, participante ou passive selon les cas, dans le cadre de travaux collectifs sur l'estive ou de réunions hors saison, sur seize estives représentatives de la diversité départementale.

${ }^{6}$ Lois pastorales de 1972, indemnité compensatoire de handicaps naturels (ICHN), prime au maintien des systèmes d'élevage extensifs (PMSEE), puis prime herbagère agro-environnemental (PHAE), autres mesures agrienvironnementales, aides directes (prime au maintien du troupeau de vaches allaitantes [PMTVA], prime à la brebis et à la chèvre $[\mathrm{PBC}])$, contrats territoriaux d'exploitation (CTE), puis contrats d'agriculture durable, vo-
} 
pour leur part, font l'objet d'un dynamisme exemplaire depuis une vingtaine d'années (Eychenne, 2006 ; SUAIAPyrénées, 2002) : depuis la première enquête pastorale de 1972, les surfaces pastorales ariégeoises se sont maintenues et les effectifs, exprimés en $\mathrm{UGB}^{7}$, ont augmenté de près de $30 \%$, notamment grâce à l'accueil de troupeaux extérieurs. Ce phénomène d'ouverture des estives aux éleveurs non-usagers (Encadré 1) est connu et référencé de longue date (Fédération pastorale de l'Ariège, 1997), il a fait l'objet d'approches techniques dans le cadre des programmes d'amélioration pastorale (quels sont les besoins et les attentes spécifiques de ces éleveurs transhumants). Mais les dimensions géographiques du problème, tant en termes de rapport à l'espace que de rapport à la ressource, ont été négligées. Ce manque est sans doute imputable au désintérêt actuel des géographes ruraux pour les questions pastorales pyrénéennes. Elles sont pourtant d'un grand intérêt pour l'analyse de l'évolution des rapports que les éleveurs entretiennent avec la montagne, et peut-être plus encore pour l'analyse des rapports qu'ils entretiennent entre eux, autour de la montagne.

Après des années de déprise, l'estive est donc redevenue une ressource convoitée, mais la relance pastorale observée en Ariège, comme sur l'ensemble du massif pyrénéen, n'est pas le fait d'un spectaculaire redéploiement des exploitations de haute montagne (sur les zonages, voir Encadré 2), traditionnellement utilisatrices des pâturages d'altitude. Ces exploitations ont pourtant affiché une résistance supérieure aux prévisions alarmistes des années 1980, mais, confrontées à des contraintes naturelles renforcées, elles peinent à maintenir leurs troupeaux et plus encore leurs hommes ${ }^{8}$. Au contraire, les éleveurs des zones de "montagne simple», plus favorables à l'élevage (mécanisation possible, rendements fourragers plus élevés), ont pu se saisir des nouveaux soutiens mis en place par la réforme de la PAC de 1992 pour se restructurer : la prime à la vache allaitante (PMTVA) les a conduits à se spécialiser dans l'élevage bovin allaitant et à augmenter la taille de leurs troupeaux souvent au-delà des ressources disponibles sur les sièges d'exploitation ${ }^{9}$. Dans ces zones traditionnellement sédentaires d'élevage laitier ou de veaux sous la mère, le recours à l'estive s'est développé grâce à l'ouverture mise en place dans le cadre de la relance pastorale. L'instauration de la prime à l'herbe (PMSEE, puis PHAE) obtenue sur les surfaces collectives

lets pastoraux des contrats de plan État-région (CPER) et des conventions de massif, etc.

7 Unité de gros bétail : 1 vache $=1$ UGB, 1 brebis $=0,15$ UGB.

${ }^{8}$ La zone de haute montagne a perdu $10 \%$ des UGB totales et $62 \%$ des exploitations entre 1979 et 2000 .

9 Sur la zone de montagne simple, les UGB totales ont augmenté de $12 \%$ entre 1979 et 2000 : les vaches allaitantes ont augmenté de $74 \%$, les vaches laitières ont diminué de $70 \%$. Le nombre d'exploitations a diminué de $53 \%$.
Encadré 1. Propriété des estives et droits d'usage en Ariège

En Ariège, les surfaces d'altitude relèvent principalement de formes de propriété collective : domaniale (43\%), communale ( $46 \%$ ) ou privée collective (5\%), la propriété privée individuelle ne concernant que $6 \%$ des surfaces. L'accès aux pâturages d'altitude est généralement régulé par le respect de droits d'usage plus ou moins strictement codifiés : sur terrains domaniaux, le Code forestier régit l'ensemble des droits des usagers; sur les terrains communaux ou privés, les droits peuvent être minutieusement décrits par des chartes d'Ancien Régime, ou définis de façon plus ou moins précise par les usages locaux (et généralement oraux). Au sens strict, les droits d'usage correspondent à l'autorisation accordée à une communauté usagère de prélever une ressource (ici pastorale) sur des terrains ne lui appartenant pas. Il s'agit donc d'une forme ancienne d'aliénation de la propriété privée. Le Code forestier ne précise pas les critères de définition des éleveurs usagers, il ne reconnaît que des communes usagères (celles dont les droits ont été reconnus comme fondés au 31 juillet 1827). En Ariège, la norme généralement admise conduit à reconnaître comme usagers (ou ayants droit) les éleveurs dont le siège d'exploitation est situé sur une commune usagère, qui y vivent plus de 6 mois par an (la «cheminée qui fume»), y paient des impôts, les bêtes usagères devant, de plus, hiverner sur la commune. Cette définition est le plus souvent reprise pour définir les usagers des «montagnes » communales ou privées qui sont soumises à droits d'usage. Les éleveurs utilisant les pâturages collectifs sans bénéficier de droits d'usage sont qualifiés d'éleveurs non-usagers ou, plus souvent, d'étrangers.

a renforcé leur attractivité, le coût direct de l'estive en étant réduit d'autant ${ }^{10}$.

Le pastoralisme ariégeois a donc changé de visage. Dans ce pays à tradition d'estivage, associant de façon continue à l'intérieur des vallées de haute montagne différents terroirs complémentaires (fonds de vallée, zones intermédiaires, estive), l'arrivée des éleveurs non-usagers a conduit au développement d'une véritable transhumance de proximité, fondée sur la déconnexion spatiale des terres d'hivernage et d'estivage, fût-ce à l'échelle de quelques dizaines de kilomètres. Le mouvement de relance pastorale, en introduisant en estive des hommes, des techniques et un rapport à l'espace "étrangers » au système local, est sans conteste un élément perturbateur tant des représentations que des pratiques de gestion du territoire pastoral. La dimension strictement collective $\mathrm{du}$ pastoralisme pyrénéen conduit dès lors à s'interroger sur les arrangements à l'œuvre sur les "montagnes », entre les éleveurs « d'en haut » et ceux « d'en bas » ${ }^{11}$, et à rechercher si les confrontations de pratiques, de représentations, d'ancrage pastoral aboutissent à une «représentation

\footnotetext{
10 En Ariège, le coût de l'estive, hors transport, se situe le plus souvent entre 20 et 60 euros par UGB; pour les éleveurs qui en bénéficient, la prime à l'herbe couvre généralement de 50 à $100 \%$ de ce coût.

11 Les éleveurs de haute montagne sont désormais minoritaires sur les estives ariégeoises.
} 


\section{Encadré 2. Les zonages «montagne»}

Les zones de « haute montagne » et de "montagne simple» évoquées dans cet article renvoient au zonage institutionnel établi pour le paiement des indemnités compensatoires de handicaps naturels (directive 75/268/CEE du 28 avril 1975). En France, la zone de montagne (souvent nommée «montagne simple », pour éviter les confusions) est définie à partir de caractères physiques de pente et d'altitude (altitude minimale de 600 à $800 \mathrm{~m}$, selon les zones, sur plus de $80 \%$ du territoire communal et/ou pente moyenne supérieure à $20 \%$ ou combinaison des deux facteurs). La zone de haute montagne a été, pour sa part, définie sur la base de caractères physiques accrus (altitude moyenne pondérée supérieure à $1200 \mathrm{~m}$ ) et du caractère extensif de l'élevage (densité du cheptel inférieure à $20 \mathrm{UGB} / \mathrm{km}^{2}$ ). En Ariège, la zone de haute montagne correspond schématiquement aux hautes vallées organisées selon le schéma traditionnel d'utilisation de l'espace montagnard cher aux géographes; elle concerne 471 exploitations et 18500 ha de SAU (surface agricole utile) (source : Recensement agricole 2000). La zone de montagne simple regroupe les zones nord et prépyrénéennes au relief atténué et regroupe 1364 exploitations sur 53400 ha de SAU. La quasi-totalité des unités pastorales et la plus grande partie des communes usagères (Encadré 1) se situent en zone de haute montagne.

collective de la gestion de ce milieu [et si] cette représentation collective est un élément de la reproduction sociale » (Mathieu, citée par Friedberg, 1992).

Il apparaît clairement que ce nouveau «noyau » transhumant est à l'origine d'une nouvelle forme de relation à la ressource pastorale $d^{\prime}$ abord perçue comme une opportunité fourragère permettant de conforter la reconversion et l'agrandissement. Les pâturages d'altitude sont alors envisagés comme une ressource d'appoint réservée aux animaux les moins exigeants ou les moins prometteurs. On est donc loin des systèmes strictement pastoraux développés par les éleveurs de haute montagne, pour lesquels la conduite de l'élevage est au contraire entièrement organisée autour de la pratique d'estive, considérée comme une ressource essentielle dans une organisation territoriale structurée de façon continue à l'échelle de la vallée.

"C'est notre richesse, moi je parle des zones de montagne, les cantons, tous les cantons de montagne, [...], c'est notre seule richesse, [...], moi je dirais, ces gens-là ils peuvent travailler d'une autre façon, parce que tout le monde n'estive pas, dans la plaine, on peut se permettre. Nous on peut pas faire autrement, priorité à nous, et puis les autres. Eux ils ont la chance, ils ont tout plat, ils ont des structures d'un seul tenant, ceci, cela, c'est leur richesse, ils peuvent faire des céréales [...]. Nous on peut pas, nous la seule richesse qu'on a c'est l'estive, qu'on puisse en profiter le plus possible. »

Pour les éleveurs transhumant depuis les zones préet nord-pyrénéennes, en effet, l'estive est totalement déconnectée des étages inférieurs. Le système fourrager est donc fondé sur l'articulation de surfaces complémentaires mais émiettées, en archipel. Les animaux montent en camion directement sur la «montagne » et les dynamiques affectant les zones intermédiaires ou les fonds de vallée leur sont totalement étrangères. Alors que, pour les éleveurs usagers, l'estive participe d'une inscription territoriale vécue à l'échelle de la commune, ou de la vallée, et renforcée par la dimension historique des droits d'usage ; il s'agit d'abord, pour les éleveurs transhumants, d'une ressource fourragère d'autant moins appropriée qu'ils peuvent en changer au gré des opportunités, voire envisager l'abandon de la transhumance.

"Si j'avais la place, je garderais les bettes en bas, tout ce que je peux garder en bas, je le garde, d'un je les ai plus sous les yeux, et de deux, au portefeuille, la marchandise est pas la même. J'aurais de la place en bas, des vaches qui ont pas de veaux, ou des génisses, tout ça, je les mettrais à la montagne, mais des veaux..."

"S'il y avait la place par en bas de les y mettre encore mieux... Pour les vaches, si j'avais la place elles resteraient en bas. [...] Non, pas pour les pertes mais, les veaux, s'ils avaient une bonne place avec de la bonne herbe, ils seraient aussi bien par là l'été que d'aller roder sur les montagnes, ils seraient plus jolis ici que là-haut. »

Le lien entre l'éleveur et l'estive est donc de plus en plus ténu, dans le temps et dans l'espace ${ }^{12}$. Cette évolution est à l'origine d'une méconnaissance des territoires pastoraux par les éleveurs utilisateurs. Bien qu'ils fassent généralement preuve d'une bonne connaissance topographique de leur "montagne », puisqu'ils doivent la parcourir en tous sens afin de "ramasser » les animaux pour les soins ou en fin de saison, les éleveurs laissent en effet apparaître dans leurs discours un relatif désintérêt, voire une connaissance très approximative de la valeur pastorale des pâturages d'altitude parcourus par leurs troupeaux. Bien sûr, il y a de «bonnes » et de «mauvaises » montagnes, selon la qualité de la ressource fourragère liée notamment à l'altitude. Mais c'est à travers la valorisation qui en est faite par l'animal que cette qualité est estimée. Pour les éleveurs estivant, la «montagne » n'existe que médiatisée par les animaux, sa valeur est donc appréciée à partir de l'état des troupeaux à la descente d'estive. Dans un contexte de diminution des troupeaux usagers, les normes techniques régissant l'usage des pâturages d'altitude s'attachent d'abord à définir le nombre d'animaux que la «montagne» peut supporter sur la saison, et donc le nombre d'animaux «étrangers » qui peuvent être introduits. Il n'est que rarement question (y compris lors des entretiens avec les pâtres) d'itinéraires strictement définis à partir d'une connaissance précise de la valeur pastorale des différents quartiers de l'estive. Ainsi, la flore est

\footnotetext{
${ }^{12}$ La moitié des éleveurs enquêtés dans le cadre de ma thèse, usagers comme non-usagers, ne s'inscrit pas dans une tradition familiale d'estivage. Parmi les autres, près de la moitié évoque un changement de "montagne » depuis moins de vingt ans, et beaucoup n'excluent pas de nouveaux changements. Finalement, parmi les éleveurs que j'ai rencontrés, un tiers seulement évoque la continuité d'une pratique sur une estive donnée à l'échelle de la mémoire familiale.
} 
largement méconnue, et les éleveurs ne mobilisent dans leurs discours que quelques espèces emblématiques, telle la fameuse réglisse (trifolium alpinum), dont tout le monde parle mais que beaucoup ne reconnaissent pas. La connaissance fine de la montagne dont pouvaient se prévaloir les « anciens », indispensable pour garantir le renouvellement de la ressource lors des périodes de surcharge pastorale, se transmet désormais sous forme d'histoires, de mythes, dont la base technique reste obscure. Ainsi en est-il de la « rudesse » de l'herbe, élément essentiel de caractérisation de certaines estives de moyenne altitude.

"L'herbe est rude, je pense que c'est assez rare d'ailleurs d'être aussi rude. $Y$ en a qui vous l'expliqueront mieux que moi, si elles $n^{\prime} y$ ont pas tété, elles dépérissent, voire mourir, elles pissent le sang, comme on $\mathrm{dit}^{13}$. "

"L'herbage est rude, les bêtes qui sont pas habituées, vous avez vite fait de faire des enterrements. »

La qualité de l'herbe est d'abord une question de force, elle est dure ou rude, ou douce. Et la rudesse de l'herbage confère aux vaches qui sont habituées (et donc à leurs éleveurs) une supériorité incontestable, des qualités de vaillance et de robustesse qui certifient leur caractère montagnard. Et la légende résiste aux faits. En effet, compte tenu de l'admission de nouveaux troupeaux, des changements d'estive, des achats d'animaux à l'extérieur de l'exploitation, certains éleveurs se trouvent amenés à transgresser la norme (" on ne peut mettre sur des montagnes rudes que des vaches qui y ont tété ») et constatent que les animaux s'adaptent très bien.

"Je crois que c'est aussi une légende la rudesse de l'herbe, je sais pas si c'est parce qu'on y a fait des aménagements de débroussaillage, que c'est plus pacagé qu'avant, ou que les vaches sont plus habituées aussi à la rude, avant, les gens, ils se les chouchoutaient chez eux et quand elles arrivaient là-haut... »

Les discours des éleveurs mettent ainsi en évidence l'absence d'adaptation des référentiels techniques pastoraux à l'évolution des pratiques, d'une part, des connaissances savantes, d'autre part.

Tout se passe comme si le système de normes élaboré par les éleveurs estivant remplissait davantage des fonctions de structuration du groupe social, à travers le partage de représentations communes, que des fonctions strictement opératoires sur le plan technique. En effet, la diversification des systèmes pastoraux a conduit à la confrontation et à la mise en concurrence des cadres de

\footnotetext{
${ }^{13}$ Les entretiens réalisés auprès de vétérinaires intervenant en estive ne permettent pas de faire un lien entre ce mythe de l'herbe rude et une pathologie précise. On peut évoquer des pistes : piroplasmose (plus fréquente sur les estives réputées rudes), intoxication par la fougère aigle que les animaux peuvent consommer en période de disette (à rapprocher du surchargement pastoral du maximum démographique) ou problème de transition alimentaire quand les animaux passaient très rapidement de l'étable à l'estive...
}

référence techniques entre les éleveurs «d'en-haut » et les éleveurs "d'en-bas », notamment autour des modalités de conduite des troupeaux. Le caractère collectif du pastoralisme pyrénéen rend ces questions particulièrement prégnantes, puisque, durant la saison d'estive, les choix individuels sont subordonnés à l'intérêt général, notamment autour des questions sanitaires (le mélange des troupeaux augmente les risques de contagion) et de conduite de la reproduction (les mâles sont mis en commun). Les normes régissant la conduite des troupeaux sur l'estive jouent donc ici un rôle de toute première importance pour garantir le contrôle du groupe sur toute velléité d'innovation individuelle. Dans ce contexte, les éleveurs usagers s'attachent à maîtriser l'introduction éventuelle de nouvelles variantes techniques. L'hégémonie de la race bovine gasconne sur les estives ariégeoises est à ce titre particulièrement éclairante ${ }^{14}$. Les règles conditionnant le choix des reproducteurs mâles fixent la hiérarchie entre races (et, de fait, entre éleveurs). N'accepter que des taureaux gascons, $c^{\prime}$ est une manière symbolique d'affirmer la suprématie de cette race en estive, sa légitimité face à des races bouchères qui ont failli causer sa disparition. Et puisque « la Gasconne, c'est le pays, c'est aussi simple que ça », les éleveurs de montagne peuvent ainsi réaffirmer leur propre légitimité, leur appropriation des espaces d'altitude face aux éleveurs et aux systèmes d'exploitation de plaine et de piémont.

\section{Une certaine façon d'être éleveur : une redéfinition de l'entre-soi}

La hiérarchie symbolique entre éleveurs est confirmée de façon très pragmatique par le contrôle précis que les éleveurs usagers exercent sur l'ouverture des estives aux éleveurs «étrangers ». L'attachement aux droits d'usage hérités de l'Ancien Régime permet de circonscrire strictement les contours du groupe « légitime » aux éleveurs ayants droit, détenteurs de ce fait d'un certain capital social. Les droits d'usage permettent donc de maintenir les stratifications sociales sur l'estive, d'imposer des normes techniques adaptées au système traditionnel d'estivage, mais aussi de maintenir l'accès aux estives en dehors du système marchand en limitant les droits des propriétaires et en interdisant de fait toute concurrence entre éleveurs ou entre usages. L'accès des "montagnes » est donc régi par des règles très inégalitaires ${ }^{15}$ et toute

\footnotetext{
${ }^{14}$ Il s'agit là d'une spécificité départementale. Dans les autres départements de la chaîne, les troupeaux sont beaucoup moins spécialisés dans une race, et les taureaux sont souvent de race charolaise pour pouvoir être utilisés en « croisement industriel » sur toutes les vaches.

${ }^{15}$ Les cotisations sont souvent deux fois plus élevées pour les éleveurs "étrangers », qui sont aussi contraints la plupart du temps à limiter le nombre d'animaux « amontagnés ».
} 
nouvelle admission est soumise à un système complexe de parrainage et de mise à l'épreuve.

"Il est jeune mais moi j'ai fait un peu de jeunesse avec sa mère et son père, ça fait qu'on se connaît. C'est des vieilles familles, des vieilles familles qu'on se connaît, c'est moi qui avais téléphoné. Disons que c'est plus prudent de rentrer quelqu'un qu'on connaît que non pas. Il y a des annonces sur le 09 de gens qui cherchent, mais on peut pas rentrer quelqu'un comme ça.»

"Les non-usagers, c'est toujours des amis d'usagers, qui les ont fait monter, à partir du moment où il y a de la place..."

"De toute façon à la montagne vous ne pouvez jamais entrer que par connaissance. C'est très rare les montagnes où vous avez une annonce pour placer... »

Bien qu'il soit justifié par son historicité, l'attachement à un système inégalitaire $\mathrm{d}^{\prime}$ accès aux estives relève avant tout d'une problématique fonctionnelle adaptée aux modalités actuelles de gestion des surfaces collectives. "Il ne s'agit pas de plaquer le présent sur le passé mais de trouver dans celui-ci l'esquisse de solutions que nous croyons justes aujourd'hui, non parce qu'elles ont été pensées hier, mais parce que nous les pensons maintenant » (Pouillon, 1975). En effet, ce système de cooptation permet aux éleveurs de haute montagne de maintenir une part de l' « entre-soi » fragilisé par la nécessaire ouverture des estives aux troupeaux d'aval. On peut ainsi identifier différents gradients de légitimité selon des cercles concentriques d'appartenance territoriale : le canton, la zone de montagne, le département. Et si, sur la montagne, "il y en a qui sont plus étrangers que d'autres », les seuls vrais étrangers que tout le monde redoute sont ceux qui viennent des départements voisins, suspectés d'introduire une forme de spéculation sur les surfaces d'altitude. Alors que l'Ariège ne fait pas l'objet de grands mouvements interdépartementaux, le cadre départemental sert de référence majeure aux éleveurs pour définir $l^{\prime}$ «ici » et le «nous ». L'Ariège, finalement entendue comme la montagne ariégeoise, représente donc le territoire d'appartenance des éleveurs estivant, elle joue un rôle central dans la définition d'une identité collective (nous) devant être défendue contre les prétentions des autres (les éleveurs qui n'estivent pas, mais surtout les autres utilisateurs de la montagne).

"La montagne, j'en serais jaloux. [...] C'est vrai qu'il y a des fois on voudrait se le garder pour nous [les éleveurs]. Et revenir peut-être, c'est vrai, à une autre époque. Enfin, ça c'est du rêve. »

"Non non non, tant que j'aurai des bêtes, elles iront à la montagne. Si je peux plus aller à la montagne, j'aurai plus de bêtes. [...] Si on pouvait m'interdire de montagne? Là je serais méchant, je deviendrais méchant, peut-être même con à la limite. »

La référence à la montagne permet aux éleveurs estivant, qu'ils soient «d'en haut » ou «d'en bas », de se reconnaître dans une certaine façon de pratiquer leur métier, envisagée comme une distinction. Les caractéristiques physiques de la montagne : relief, climat, végétation, parent les bêtes comme les hommes de qualités spécifiques, parmi lesquelles la ténacité et la résistance : aux conditions extrêmes du milieu, aux bouleversements de la société traditionnelle, à l'homogénéisation des modes de production, à la concurrence exercée par les autres usagers de la montagne.

Dans ce cadre, la référence à la tradition dont les éleveurs maillent leurs discours pour justifier leurs pratiques, qu'ils soient ariégeois de souche ou néoruraux, qu'ils s'inscrivent ou non dans une longue tradition familiale d'estivage, est d'abord un signe de réaffirmation collective de leur légitimité dans la gestion des ressources pastorales, liée à l'antériorité de leurs pratiques. («C'est comme ça, ça a toujours été, et c'est toujours resté comme ça et ça marche très bien. ») En effet, «l'utilité en général d'une tradition est de fournir au présent une caution pour ce qu'il est. [...] L'utilité en particulier d'une tradition est d'offrir à tous ceux qui l'énoncent et la reproduisent au jour le jour le moyen d'affirmer leur différence et, par là même, d'asseoir leur autorité » (Lenclud, 1987). Dans ce contexte, le recours à la tradition ne peut être envisagé comme un signe d'archaïsme et de résistance au changement, mais comme une forme de résistance aux prescriptions extérieures, la marque de la capacité des groupes d'éleveurs à définir eux-mêmes les modalités de leur rapport à la ressource.

\section{Conclusion}

Cette approche renouvelée des questions pastorales ouvre de nouvelles perspectives dans la compréhension des dynamiques à l'œuvre sur les espaces d'altitude. Le choix d'une analyse par les relations éleveurs-estive, envisagées du point de vue des éleveurs, permet d'aborder à la fois les dimensions matérielles et idéelles des modalités de gestion de la ressource. Cette pratique apparemment immuable qu'est l'estivage de troupeaux s'est largement adaptée à l'évolution des systèmes d'élevage. Les systèmes de pensée des éleveurs de montagne ont changé eux aussi, et la référence à la tradition permet surtout à ces derniers de maintenir certains équilibres parfois peu compatibles avec une demande sociale croissante de biens et services de nature. En se mettant au côté des éleveurs, dans l'attention portée au regard qu'ils portent eux-mêmes sur ce territoire à haute valeur symbolique, le chercheur accède à une nouvelle forme de compréhension des rapports société-nature. La relative inefficience de certaines pratiques en termes de renouvellement de la ressource pastorale n'est alors plus imputable à un manque de technicité (ou de modernité, ou de sens du collectif), mais bien au besoin de maintenir un système social stable pour des éleveurs de haute montagne par ailleurs largement fragilisés. De même, l'attachement aux droits d'usage, forme archaïque d'accès à la ressource ailleurs disparue, prend-il dans le contexte actuel une dimension 
très innovante de résistance à l'entrée des estives dans une logique concurrentielle.

\section{Références}

Balent, G., Barrué-Pastor, M., 1986. Pratiques pastorales et stratégies foncières dans le processus de déprise de l'élevage montagnard en vallée d'Oô (Pyrénées centrales), Revue géographique des Pyrénées et du Sud-Ouest, 57, 3, 403-447.

Barrué-Pastor, M., Fournié, V., 1996. La montagne ariégeoise entre friche et paysage : un consensus illusoire?, Études rurales, 141-142, 109-123.

Cavaillès, H., 1931. La Vie agricole et pastorale dans les Pyrénées des Gaves, de l'Adour et des Nestes, Paris, A. Colin.

Chevalier, M., 1956. La Vie humaine dans les Pyrénées ariégeoises, Paris, M.-Th. Génin.

Darré, J.-P., 1985. La Parole et la technique : l'univers de pensée des éleveurs du Ternois, Paris, L'Harmattan.

Darré, J.-P., Hubert, B., Lasseur, J., Landais, É., 1993. Raisons et pratiques, dialogue avec un éleveur ovin, Études rurales, 131-132, 107-181.

Di Pietro, F., Balent, G., 1997. Dynamique des pratiques pastorales et des paysages : une approche pluri-échelles appliquée aux Pyrénées ariégeoises (France), Agronomie, 17, 139-155.

Duru, M., Gibon, A., Langlet, A., Flamant, J.-C., 1979. Recherches sur les problèmes pastoraux pyrénéens, in Molénat, G., Jarrige, R. (Eds), Utilisation par les ruminants des pâturages d'altitude et parcours méditerranéens, Versailles, INRA, 231-255.

Eychenne, C., 2003. Les Éleveurs et l'estive, un regard sur l'action collective : le cas de la "montagne " ariégeoise. Thèse de doctorat en études rurales, mention géographie, Université Toulouse 2 - Le Mirail, Toulouse.
Eychenne, C., 2006. Hommes et troupeaux en montagne : la question pastorale en Ariège, Paris, L'Harmattan.

Fédération pastorale de l'Ariège, 1997. Enquête estives ariégeoises 1994, Foix.

Friedberg, C., 1992. Représentation-classification : comment $l^{\prime}$ homme pense ses rapports au milieu naturel, in Jollivet, $M$. (Ed.), Sciences de la nature, sciences de la société : les passeurs de frontières, Paris, CNRS Éditions, 357-371.

Gibon, A., 1997. Mutations des systèmes d'élevage et utilisation des espaces pastoraux privés et collectifs dans les Pyrénées centrales, in Bourbouze, A., et al. (Ed.), Pastoralisme et foncier : impact du régime foncier sur la gestion de l'espace pastoral et la conduite des troupeaux en régions arides et semi-arides, Montpellier, CIHEAM-IAMM, 69-80.

Lenclud, G., 1987. La tradition n'est plus ce qu'elle était... Sur les notions de tradition et de société traditionnelle en ethnologie, Terrain, 9, 110-123.

Mathieu, N., 1992. Géographie et interdisciplinarité : rapport naturel ou rapport interdit?, in Jollivet, M. (Ed.), Sciences de la nature, sciences de la société : les passeurs de frontières, Paris, CNRS Éditions, 129-154.

Métailié, J.-P. (Ed.), 1999. Cartographie des estives dans les Pyrénées ariégeoises : typologie et approche contemporaine, Toulouse, Géode (UMR 5602, CNRS).

Pharo, P., 1985. Savoirs paysans et ordre social : l'apprentissage $d u$ métier d'agriculteur, Paris, CEREQ.

Pouillon, J., 1975. Tradition : transmission ou reconstruction?, in Pouillon, J., Fétiches sans fétichisme, Paris, Maspéro, 155-173.

Salmona, M., 1994. Les Paysans français : le travail, les métiers, la transmission des savoirs, Paris, L'Harmattan.

SUAIA-Pyrénées, 2002. Les Exploitations pastorales pyrénéennes, Agreste Midi-Pyrénées, Aquitaine, LanguedocRoussillon, Données, 9.

Reçu le 24 août 2007. Accepté le 6 mars 2008. 